\title{
Preparation of Barium Titanate Fine Particles by Hydrothermal Method and Their Characterization
}

\author{
Satoshi WADA, Takeyuki SUZUKI and Tatsuo NOMA \\ Department of Applied Chemistry, Tokyo University of Agriculture and Technology, 24-16, Nakamachi 2-chome, Koganei-shi, Tokyo \\ 水熱法によるチタン酸バリウム微粒子の合成とそのキャラクタリゼーション \\ 和田智志・鈴木健之・野間竜男 \\ 東京農工大学工学部応用化学科, 184 東京都小金井市中町 2-24-16
}

184

[Received July 14, 1995; Accepted September 14, 1995]

\begin{abstract}
Barium titanate fine particles with average particle sizes from 20 to $90 \mathrm{~nm}$ were prepared by a hydrothermal method using titanium tetrahydroxide gel and barium hydroxide. The particle size was inversely proportional to the $\mathrm{Ba} / \mathrm{Ti}$ atomic ratio in the starting material, while it was independent of temperature in the narrow range from 70 to $150^{\circ} \mathrm{C}$. For the explanation of these findings, we proposed the following mechanism for the formation of barium titanate fine particles, i.e., the nuclei of barium titanate form via the reaction of titanium tetrahydroxide gel with barium hydroxide and then grow. In these particles, there were many lattice defects in the form of hydroxyl groups and barium vacancies, and their concentration increased with decreasing particle size. We explained this result using the model that a particle can be divided into two parts: a defective surface layer with many defects and the bulk with fewer defects. The crystal structure of barium titanate particles was cubic with an expanded lattice $(a \sim 0.402 \mathrm{~nm})$ independent of particle size. This result indicated the possibility that the lattice defects influenced the crystal structure.
\end{abstract}

Key-words : Barium titanate, Fine particles, Hydrothermal method, Size effect, Lattice defects, Crystal structure

\section{Introduction}

The size effect in the displacive-type ferroelectrics such as barium titanate, that Curie temperature $\left(T_{\mathrm{c}}\right)$ and the tetragonality $(c / a)$ rapidly decrease with decreasing particle size, has been studied by many investigators. ${ }^{1 \text {-11) }}$ However, it has never been explained although many investigators have confirmed the presence of this effect and have tried to clarify it. ${ }^{1)-13)}$ As one of the causes, the difference in the fine structure (or microstructure) of the samples according to the preparation method is noted. There are many methods for the preparation of barium titanate fine particles. Among them, the sol-gel method ${ }^{14), 15)}$ and hydrothermal method ${ }^{16)-21}$ are well known. However there is a drawback in the sol-gel method that barium carbonate forms easily because of the use of compounds that include carbon as raw materials. There is also a drawback in the hydrothermal method that lattice defects such as $\mathrm{H}_{2} \mathrm{O}$ and $\mathrm{OH}^{-}$exist in barium titanate fine particles. Thus, it is very difficult to investigate the size effect precisely using the samples prepared by these methods.

Recently, Vivekanandan et al. ${ }^{16)}$ found that $\mathrm{H}_{2} \mathrm{O}$ and $\mathrm{OH}^{-}$included in barium titanate particles with particle sizes of 200-500 nm prepared hydrothermally can contribute to the formation of a cubic phase with an expanded lattice constant, $a=0.4021 \mathrm{~nm}$, which is metastable around room temperature. Moreover, Hennings and Schreinemacher showed that a cubic phase of barium titanate fine particles with a particle size of about $200 \mathrm{~nm}$ prepared hydrothermally can change to the tetragonal phase upon the removal of $\mathrm{H}_{2} \mathrm{O}$ and $\mathrm{OH}^{-}$in the particles, without the change of particle size. ${ }^{20)}$ The above results indicated the possibility that the lattice defects could also influence the crystal structure of barium titanate fine particles, as well as the particle and grain sizes. At present, we believe that in order to explain the size effect in the displacive-type ferroelectrics it is important to investigate the effect of the lattice defects such as $\mathrm{OH}^{-}$on the crystal structure, as well as the effect of the particle sizes. Moreover it is of great interest to study the crystal structure of barium titanate fine particles with particle sizes smaller than $120 \mathrm{~nm}$.

In this study, we prepare barium titanate fine particles with particle sizes smaller than $100 \mathrm{~nm}$ formed by a hydrothermal method, and investigate the characteristics of the as-prepared particles, e.g., particle size, crystallite size, size distribution, crystal structure and lattice defects. This study is the first step in the investigation of the size effect in barium titanate fine particles on the basis of particle sizes and lattice defects.

\section{Experimental}

\subsection{Sample preparation}

Titanium tetrachloride, $\mathrm{TiCl}_{4}$ (Kishida Chem. Co., Ltd., research grade, purity $>99.9 \%$ ) and barium hydroxide, $\mathrm{Ba}(\mathrm{OH})_{2} \cdot 8 \mathrm{H}_{2} \mathrm{O}$ (Wako Pure Chem. Ind., Ltd., research grade, purity $>98 \%$ ) were used as raw materials. After dissolution of titanium tetrachlo- 
ride in cooled water, from which carbon dioxide was removed beforehand, aqueous ammonia was added slowly to this solution at temperatures below $10^{\circ} \mathrm{C}$, and then titanium tetrahydroxide, $\operatorname{Ti}(\mathrm{OH})_{4}$, gel was formed. Ammonium ions and chloride ions in this gel solution were mostly removed by repeated decantation (about 15 times), and the gel solution was used as a titanium source. After addition of barium hydroxide to the gel solution at various $\mathrm{Ba} / \mathrm{Ti}$ atomic ratios in the range from 2 to 40 , the solution was stirred in a conventional autoclave at various temperatures in the range from 50 to $150^{\circ} \mathrm{C}$ for $4 \mathrm{~h}$, and barium titanate was precipitated. This precipitate was filtered, washed, and then stirred in acetic acid solution $\left(6 \mathrm{~mol} \cdot \mathrm{dm}^{-3}\right)$ at $50^{\circ} \mathrm{C}$ for $1 \mathrm{~h}$, in order to remove unfavorable barium salts such as barium hydroxide and barium carbonate. After acetic acid treatment, the precipitate was filtered, washed, dried in vacuum overnight at temperatures from 50 to $80^{\circ} \mathrm{C}$, and used as samples.

\subsection{Characterization of the samples}

The crystal structure of the particles was investigated at room temperature using a powder X-ray diffractometer (XRD : RAD-2B and RAD-2C, Rigaku, $\mathrm{Cu} \mathrm{K} \alpha, 30 \mathrm{kV}, 20 \mathrm{~mA}$ ) and a Fourier transform infrared spectrometer (FT-IR : JIR-100, JEOL). In the measurement of the lattice constant, $\mathrm{SiO}_{2}(\alpha$-quartz) powder was used as the external standard. $\mathrm{H}_{2} \mathrm{O}$ and $\mathrm{OH}^{-}$in the particles were measured using FT-IR and by differential thermal analysis with thermogravimetry (TG-DTA : TG-DTA 2000, Mac Science). For the FT-IR measurement, we formed pellets of the samples by diluting the particles to 1 mass\% with $\mathrm{KBr}$ powder with drying at $100^{\circ} \mathrm{C}$ in vacuum overnight, and measured their FTIR spectra at room temperature in dry air. Average particle sizes were estimated using a transmission electron microscope (TEM: H-700H, Hitachi, 200 $\mathrm{kV}$ ). The $\mathrm{Ba} / \mathrm{Ti}$ atomic ratio in the particles was measured by conventional gravimetric analysis as follows. The particles were dissolved in concentrated sulfuric acid with ammonium sulfate at $50^{\circ} \mathrm{C}$. After cooling to room temperature, the slow addition of cooled water to the solution in an ice-cold water bath resulted in precipitation of barium sulfate. This precipitate was filtered, washed, dried slowly, put into a crucible, and ignited to a constant weight, which was taken to be that of barium sulfate. The slow addition of aqueous ammonia to the filtrate resulted in precipitation of titanium tetrahydroxide. The precipitate was filtered, washed, dried slowly, put into a crucible, and ignited to a constant weight, which was taken to be that of titanium dioxide.

\section{Results}

3.1 Conditions for hydrothermal processing of barium titanate fine particles

Barium titanate fine particles were prepared hydrothermally for $4 \mathrm{~h}$ with the conventional au- toclave using titanium tetrahydroxide gel and barium hydroxide as the starting materials. Figure 1 shows the conditions for the formation of barium titanate at various temperatures and $\mathrm{Ba} / \mathrm{Ti}$ atomic ratios in the starting material. In the particles prepared at temperatures higher than $70^{\circ} \mathrm{C}$ and with $\mathrm{Ba} / \mathrm{Ti}$ atomic ratios greater than 2 , all XRD peaks were assigned to barium titanate $\left(\mathrm{BaTiO}_{3}\right)$ as shown in Fig. $2(\mathrm{Ba} / \mathrm{Ti}$ atomic ratios greater than 1$)$. For conditions other than the above, only amorphous phase was observed, as shown in Fig. 2 (Ba/Ti atomic ratio of 1), which changed to titanium-rich barium titanate phase such as $\mathrm{Ba}_{2} \mathrm{Ti}_{9} \mathrm{O}_{20}$ upon thermal treatment at temperatures higher than $1000^{\circ} \mathrm{C}$.

3.2 Characterization of samples prepared at $150^{\circ} \mathrm{C}$ and with various $\mathrm{Ba} / \mathrm{Ti}$ atomic ratios from 2 to 40

\subsubsection{Particle size}

Figure 2 shows the XRD patterns of the samples

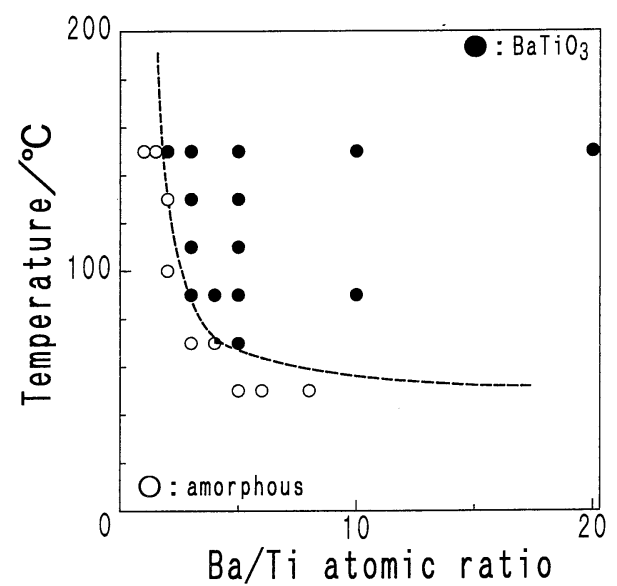

Fig. 1. The conditions for the formation of barium titanate at various temperatures with $\mathrm{Ba} / \mathrm{Ti}$ atomic ratios in the starting material.

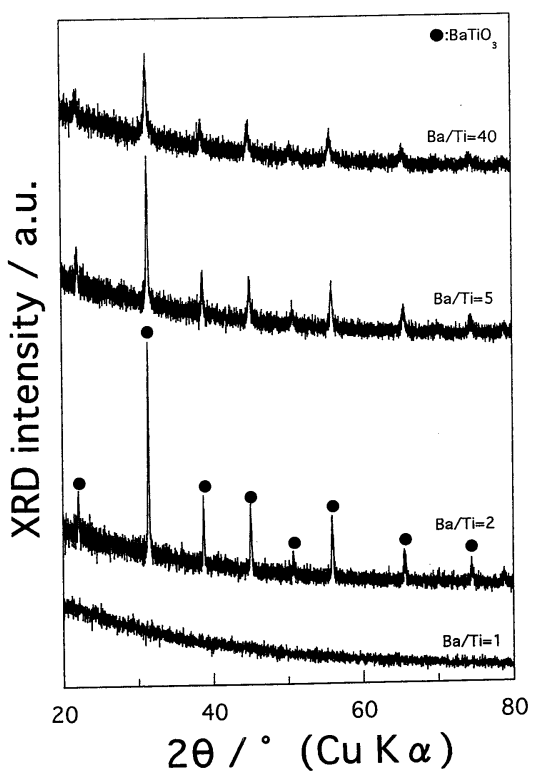

Fig. 2. The XRD patterns of the particles prepared at $150^{\circ} \mathrm{C}$ for $4 \mathrm{~h}$ with various $\mathrm{Ba} / \mathrm{Ti}$ atomic ratios. 
prepared at $150^{\circ} \mathrm{C}$ for $4 \mathrm{~h}$ with various $\mathrm{Ba} / \mathrm{Ti}$ atomic ratios. All samples prepared with $\mathrm{Ba} / \mathrm{Ti}$ atomic ratios greater than 1 were a single phase of barium titanate $\left(\mathrm{BaTiO}_{3}\right)$. The XRD intensity decreased and the peaks broadened with increasing $\mathrm{Ba} / \mathrm{Ti}$ atomic ratio in the starting material.

Figure 3 shows a selected-area electron diffraction (SAED) pattern and TEM bright-field and darkfield images of the fine particles prepared at $150^{\circ} \mathrm{C}$
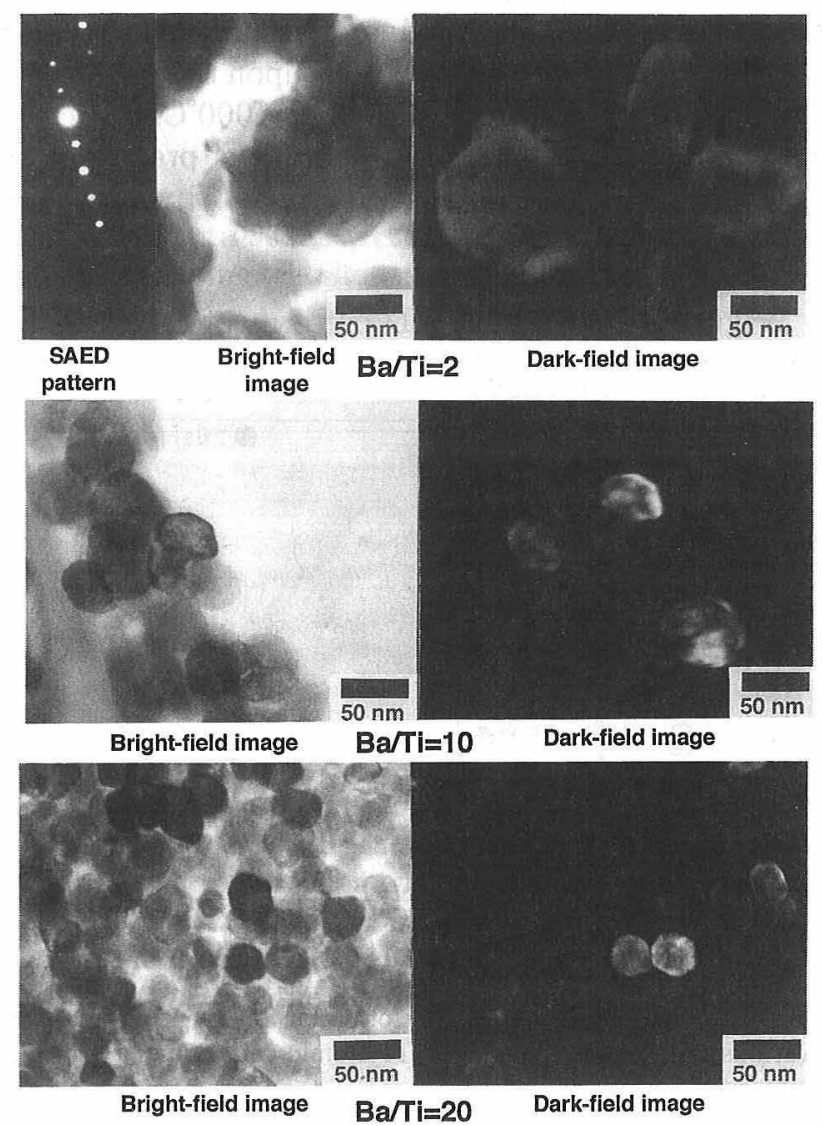

Fig. 3. A SAED pattern and the TEM bright-field and dark-field images of the particles prepared at $150^{\circ} \mathrm{C}$ for $4 \mathrm{~h}$ with various $\mathrm{Ba} /$ $\mathrm{Ti}$ atomic ratios. for $4 \mathrm{~h}$ with various $\mathrm{Ba} / \mathrm{Ti}$ atomic ratios. The average particle size decreased with increasing $\mathrm{Ba} / \mathrm{Ti}$ atomic ratio in the starting material. This result indicated that the decrease of particle sizes caused the broadening of X-ray diffraction peaks. The morphology of the particles changed from ellipsoids to spheres with increasing $\mathrm{Ba} / \mathrm{Ti}$ atomic ratio in the starting material. The SAED of a particle, as shown in Fig. 3, indicates that the particle was a single crystal with a single $\mathrm{BaTiO}_{3}$ phase. Moreover, the particle sizes in the dark-field images were in agreement with the particle sizes in the bright-field images for all samples prepared with various $\mathrm{Ba} / \mathrm{Ti}$ atomic ratios. These results also indicated that a particle was a single crystal of only barium titanate.

Figure 4 shows the particle size distributions estimated from these TEM micrographs. The particle size distribution of the sample prepared with a $\mathrm{Ba} / \mathrm{Ti}$ atomic ratio above 20 was very narrow, but the particle size distribution broadened with decreasing $\mathrm{Ba} /$ $\mathrm{Ti}$ atomic ratio in the starting material. For example, of particles with an average particle size of 89.62 $\mathrm{nm}, 70 \%$ were in the range of $89.62 \pm 20.0 \mathrm{~nm}$, and of particles with an average particle size of 19.72 $\mathrm{nm}, 95 \%$ were in the range of $19.72 \pm 5.0 \mathrm{~nm}$. Figure 5 shows the dependence of the average particle size estimated from TEM micrographs on the $\mathrm{Ba} / \mathrm{Ti}$ atomic ratio in the starting material. The average particle size decreased with increasing $\mathrm{Ba} /$ $\mathrm{Ti}$ atomic ratio in the starting material, and above the $\mathrm{Ba} / \mathrm{Ti}$ atomic ratio of 20 , the particle size was almost constant at about $20 \mathrm{~nm}$.

\subsubsection{Lattice defects}

Figure 6 shows the FT-IR spectrum of the particles prepared at $150^{\circ} \mathrm{C}$ for $4 \mathrm{~h}$ with the $\mathrm{Ba} / \mathrm{Ti}$ atomic ratio of 2 . The FT-IR spectra in this study were measured at room temperature in dry air, as mentioned above. A broad absorption band in a wide range from 2800 to $3600 \mathrm{~cm}^{-1}$ indicated the presence of considerable amounts of $\mathrm{H}_{2} \mathrm{O}$ and $\mathrm{OH}^{-}$ in the particles. ${ }^{16), 20)}$ In particular, the sharp absorp-

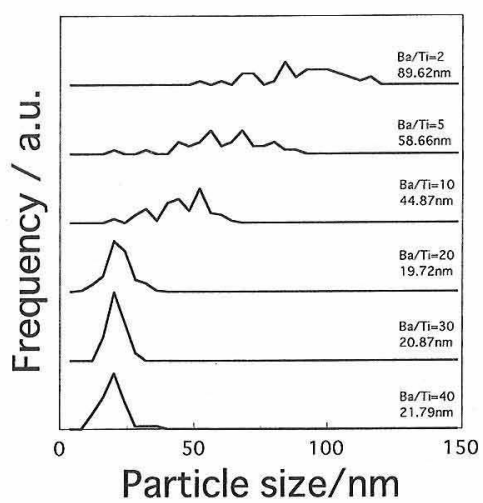

Fig. 4

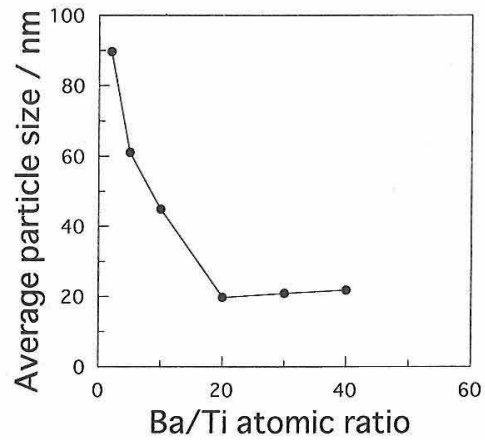

Fig. 5

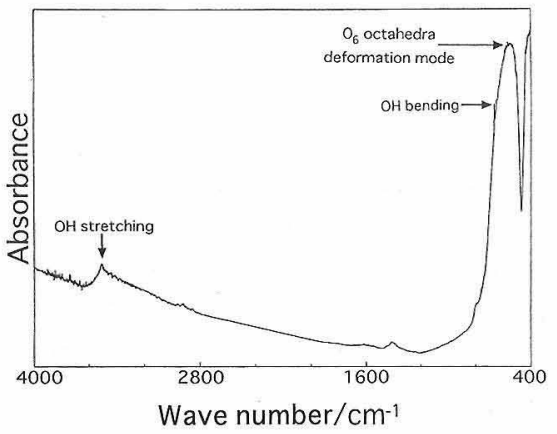

Fig. 6

Fig. 4. The particle size distributions of the particles prepared at $150^{\circ} \mathrm{C}$ for $4 \mathrm{~h}$ with various $\mathrm{Ba} / \mathrm{Ti}$ atomic ratios.

Fig. 5. The dependence of the average particle size on $\mathrm{Ba} / \mathrm{Ti}$ atomic ratio in the starting material.

Fig. 6. The FT-IR spectrum of the particles prepared at $150^{\circ} \mathrm{C}$ for $4 \mathrm{~h}$ with $\mathrm{Ba} / \mathrm{Ti}$ atomic ratio of 2 . 
tion band at around $3500 \mathrm{~cm}^{-1}$ was assigned to the stretching vibration of the hydroxyl group with an intramolecular hydrogen bond,23) which shows the presence of hydroxyl group in barium titanate lattice. The weak sharp absorption band observed at around $650 \mathrm{~cm}^{-1}$ was assigned not to $\mathrm{H}_{2} \mathrm{O}$ but to the bending vibration of the lattice hydroxyl group, as reported by Vivekanandan et al., ${ }^{16)}$ which supported the presence of the lattice hydroxyl group.

The absorption band observed at around $550 \mathrm{~cm}^{-1}$ was assigned to the bending vibration of an $\mathrm{O}_{6}$ octahedron $\left(=\mathrm{O}_{6}\right.$ octahedra deformation mode), which was assigned by Slater ${ }^{24)}$ and confirmed by Spitzer et al. ${ }^{25)}$ This band in cubic barium titanate is 3 -fold degenerate $\left(=T_{1 \mathrm{u}}\right.$ mode) because of equivalent vibrations along three axes $(a=b=c)$, but the band in pseudocubic barium titanate with slight tetragonality $(c / a>1)$ can split into two bands $\left(=A_{1}\right.$ and $E$ modes) because of the difference between $\mathrm{O}-\mathrm{O}$ lengths along the $a$-axis $(a=b)$ and $c$-axis. Thus by investigating the change of the shape and wave number of the band, information about the crystal structure and $\mathrm{O}-\mathrm{O}$ length can be obtained.

Moreover, as shown in Fig. 6, no band other than those described above was observed, which indicated that there was no impurity except for the hydroxyl group in the particles. Thus, in this study, we considered only the lattice hydroxyl group and $\mathrm{H}_{2} \mathrm{O}$ as impurities.

Figure 7 shows the absorption bands for the stretching vibrations of $\mathrm{H}_{2} \mathrm{O}$ and $\mathrm{OH}^{-}$in the particles prepared at $150^{\circ} \mathrm{C}$ for $4 \mathrm{~h}$ with various $\mathrm{Ba} / \mathrm{Ti}$ atomic ratios in the starting material. The amounts of $\mathrm{H}_{2} \mathrm{O}$ and $\mathrm{OH}^{-}$included in the particles increased with increasing $\mathrm{Ba} / \mathrm{Ti}$ atomic ratio in the starting material. Furthermore these broad bands could be separated into (1) a small sharp shoulder band for the lattice hydroxyl group ${ }^{16), 23)}$ at around $3500 \mathrm{~cm}^{-1}$ and (2) a large broad band for the surface-adsorbed hydroxyl group at around $3400 \mathrm{~cm}^{-1}$. The absorbance of the band for the surface-adsorbed hydroxyl group increased with increasing $\mathrm{Ba} / \mathrm{Ti}$ atomic ratio in the starting material, while that of the band for the lattice hydroxyl group was almost constant regardless of the $\mathrm{Ba} / \mathrm{Ti}$ atomic ratio.

We measured the amount of water included in the particles by TG measurements, and determined $\mathrm{Ba}$ / $\mathrm{Ti}$ atomic ratios in the particles by gravimetric analysis, as shown in Table 1 . All $\mathrm{Ba} / \mathrm{Ti}$ atomic ratios in these samples were less than 1 , which indicated that there were numerous barium deficiencies in the particles (we consider the deficient barium to be equivalent to the barium vacancies $V_{\mathrm{Ba}}{ }^{\prime \prime}$ in the barium titanate lattice). The number of barium vacancies increased with decreasing particle size. The amount of water ( $\sim$ hydroxyl group) also increased with decreasing particle size. These results indicated that the number of barium vacancies was corresponded to that of hydroxyl groups.

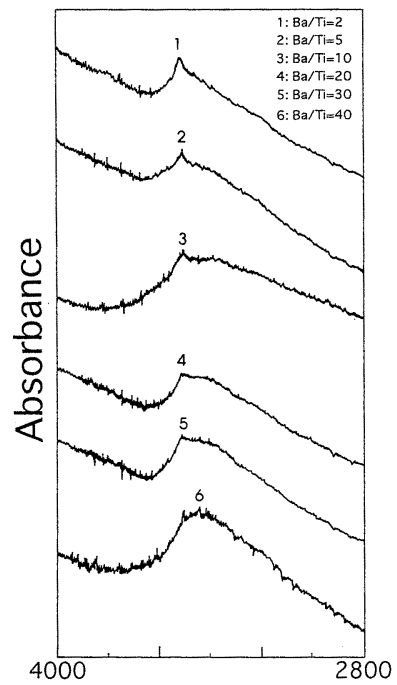

\section{Wave number $/ \mathrm{cm}^{-1}$}

Fig. 7. The FT-IR spectra for the stretching vibration of hydroxyl groups in the particles prepared at $150^{\circ} \mathrm{C}$ for $4 \mathrm{~h}$ with various $\mathrm{Ba} / \mathrm{Ti}$ atomic ratios.

Table 1. The Characteristics of Hydrothermal Barium Titanate Fine Particles Prepared at $150^{\circ} \mathrm{C}$ for $4 \mathrm{~h}$ with Various Ba/Ti Atomic Ratios in the Starting Material

\begin{tabular}{|c|c|c|c|c|}
\hline $\begin{array}{c}\mathrm{Ba} / \mathrm{Ti} \text { atomic ratio } \\
\text { in the starting } \\
\text { material }\end{array}$ & $\begin{array}{l}\text { Average } \\
\text { particle size } \\
/ \mathrm{nm}\end{array}$ & $\begin{array}{l}\text { Crystal } \\
\text { structure }\end{array}$ & $\begin{array}{c}\text { Amount } \\
\text { of water } / \%\end{array}$ & $\begin{array}{l}\mathrm{Ba} / \mathrm{Ti} \text { atomic ratio } \\
\text { in the sample }\end{array}$ \\
\hline 2 & 89.62 & cubic & 2.7 & $0.93 \pm 0.01$ \\
\hline 5 & 58.66 & cubic & 2.8 & $0.92 \pm 0.01$ \\
\hline 10 & 44.87 & cubic & 3.6 & $0.91 \pm 0.01$ \\
\hline 20 & 19.72 & cubic & 4.1 & $0.88 \pm 0.01$ \\
\hline 30 & 20.87 & cubic & - & - \\
\hline 40 & 21.79 & cubic & - & - \\
\hline $\begin{array}{l}\text { Lattice constant } \\
\text { a-axis / nm }\end{array}$ & \multicolumn{2}{|c|}{$\begin{array}{c}\mathrm{O}_{6} \text { octahedra } \\
\text { deformation mode } \\
/ \mathrm{cm}^{-1}\end{array}$} & & \\
\hline 0.4021 & \multicolumn{2}{|l|}{554} & & \\
\hline 0.4021 & \multicolumn{2}{|l|}{558} & & \\
\hline 0.4021 & \multicolumn{2}{|l|}{555} & & \\
\hline 0.4022 & \multicolumn{2}{|l|}{561} & & \\
\hline .0 .4022 & \multicolumn{2}{|l|}{563} & & \\
\hline 0.4022 & \multicolumn{2}{|l|}{559} & & \\
\hline
\end{tabular}

\subsubsection{Crystal structure}

Figure 8 shows the XRD patterns for a (200) plane of barium titanate in the particles prepared with various $\mathrm{Ba} / \mathrm{Ti}$ atomic ratios in the starting material. The peak of a (200) plane of the sample prepared with $\mathrm{Ba} / \mathrm{Ti}$ atomic ratio of 2 was separated into two peaks: (1) a large peak at low $2 \theta$ and (2) a small shoulder at high $2 \theta$, attributable to $\mathrm{Cu} \mathrm{K} \alpha_{1}$ and $\mathrm{Cu} \mathrm{K} \alpha_{2}$, respectively. However, the peaks of a (200) plane of the samples prepared with $\mathrm{Ba} / \mathrm{Ti}$ atomic ratios greater than 2 did not split. This was because the influence of particle size on line-broadening increased with increasing $\mathrm{Ba} / \mathrm{Ti}$ atomic ratio, and consequently the full width at half maximum $(=\mathrm{FWHM}), \boldsymbol{\beta}$, of the (200) plane increased with decreasing particle size. Therefore, all (200) planes in Fig. 8 exhibited no splitting for (002) and (200) 
planes, and this indicated that the crystal structure was cubic. Moreover, the angle at the maximum intensity of the peak $(2 \theta)$ was independent of particle size. The lattice constant $a$-axis estimated from $2 \theta$ was about $0.402 \mathrm{~nm}$ which is much greater than that $(0.4005 \mathrm{~nm})$ obtained by the extrapolation of the $a$ axis of the cubic phase, which is stable above $T_{c}$, to room temperature. ${ }^{26)}$ This indicated that the crystal structure of the particles was cubic with a considerably expanded lattice.

Figure 9 shows the Wilson-Hall plots for these samples, from which we determine whether these samples have lattice strain $(\boldsymbol{\eta})$ or not. These plots were measured using only the diffraction peaks by $\mathrm{Cu} \mathrm{K} \alpha_{1}$ separated using Rachinger's method. The Wilson-Hall equation used in this study is

$$
\beta \cdot \cos \theta / \lambda=1 / D+2 \eta \cdot \sin \theta / \lambda
$$

There was no lattice strain $(\boldsymbol{\eta} \sim 0)$ in these particles since the slopes of the lines were 0 , and the crystallite sizes $D$ obtained from the intersections with the vertical axis agreed, within the range of $d \pm 15 \mathrm{~nm}$, with average particle sizes $d$ estimated from TEM, i.e., $D=72.9 \mathrm{~nm}$ and $d=89.62 \mathrm{~nm} ; D=44.4 \mathrm{~nm}$ and $d=44.87 \mathrm{~nm}$; and $D=31.2 \mathrm{~nm}$ and $d=19.72 \mathrm{~nm}$ with $\mathrm{Ba} / \mathrm{Ti}$ atomic ratios of 2,10 and 20 , respectively. Moreover, this result $(D \sim d \pm 15)$ supported the result of TEM that a particle is a single crystal.

Figure 10 shows the absorption bands for the bending vibrations of $\mathrm{O}_{6}$ octahedra for the particles prepared with various $\mathrm{Ba} / \mathrm{Ti}$ atomic ratios in the starting material. The shape of the $\mathrm{O}_{6}$ octahedra deformation mode was almost symmetric ( $T_{1 \mathrm{u}}$ mode) in all samples, based on which the crystal structure of these particles was assigned as cubic. This result was also in agreement with the result in
Fig. 8 that the crystal structure was cubic. Moreover, the maximum wave number of the $\mathrm{O}_{6}$ octahedra deformation mode was almost constant regardless of particle size. This result indicates that $\mathrm{O}-\mathrm{O}$ bond length in the $\mathrm{O}_{6}$ octahedra is almost independent of particle size, which agreed with the result in Fig. 8 that the lattice constant $a$-axis is almost constant in spite of the particle size.

\subsubsection{Characteristics of particles}

The characteristics of the particles prepared at $150^{\circ} \mathrm{C}$ for $4 \mathrm{~h}$ with various $\mathrm{Ba} / \mathrm{Ti}$ atomic ratios in the starting material are described as follows.

(1) A particle is a single crystal of a barium titanate.

(2) The particle size decreases with increasing $\mathrm{Ba} / \mathrm{Ti}$ atomic ratio in the starting material, and are almost constant at about $20 \mathrm{~nm}$ above the $\mathrm{Ba} / \mathrm{Ti}$ atomic ratio of 20 .

(3) The crystal structure of the particles is cubic with an expanded lattice.

(4) There are two kinds of hydroxyl groups in the particles: the surface-adsorbed hydroxyl group, the amount of which increases with decreasing particle size, and the lattice hydroxyl group, the amount of which remains almost constant regardless of the particle size.

(5) There are many barium vacancies in the particles, and the concentration of barium vacancies increases with decreasing particle size.

\subsection{Characterization of samples prepared at various temperatures from 70 to $150^{\circ} \mathrm{C}$ with $\mathrm{Ba} / \mathrm{Ti}$ atomic ratio of 5}

Unlike the above results, for the particles prepared with a constant $\mathrm{Ba} / \mathrm{Ti}$ atomic ratio at various temperatures from 70 to $150^{\circ} \mathrm{C}$, the particle size, the

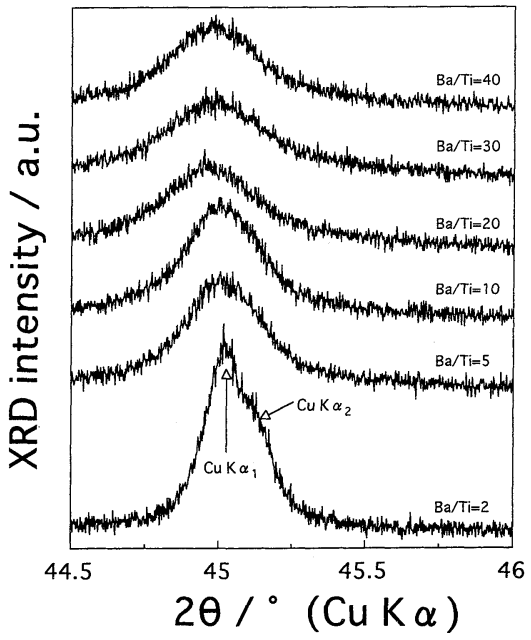

Fig. 8

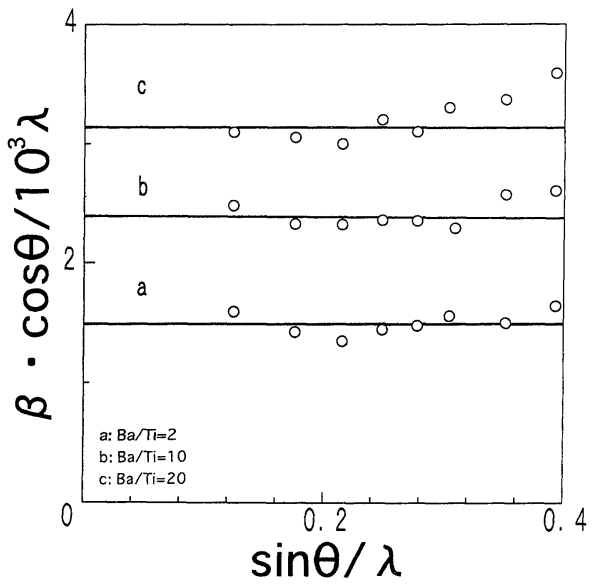

Fig. 9

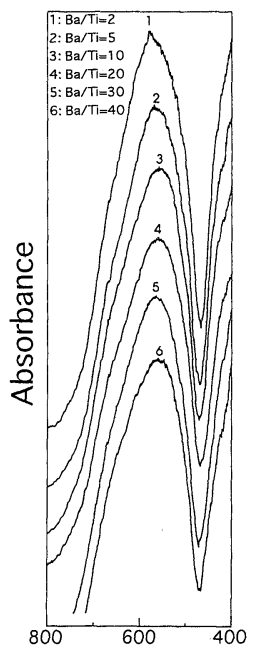

Wave number $/ \mathrm{cm}^{-1}$

Fig. 10

Fig. 8. The XRD patterns for a (200) plane of barium titanate in the particles prepared at $150^{\circ} \mathrm{C}$ for $4 \mathrm{~h}$ with various $\mathrm{Ba} / \mathrm{Ti}$ atomic ratios. Fig. 9. The Wilson-Hall plots for the particles prepared at $150^{\circ} \mathrm{C}$ for $4 \mathrm{~h}$ with various $\mathrm{Ba} / \mathrm{Ti}$ atomic ratios.

Fig. 10. The FT-IR spectra for the bending vibration of $\mathrm{O}_{6}$ octahedra in the particles prepared at $150^{\circ} \mathrm{C}$ for $4 \mathrm{~h}$ with various $\mathrm{Ba} / \mathrm{Ti}$ atomic ratios. 
fine structure, and lattice constants were almost constant, which indicates that the characteristics of the particles prepared in this study were greatly dependent on the $\mathrm{Ba} / \mathrm{Ti}$ atomic ratio in the starting material but almost independent of temperature. Therefore, below, we discuss the mechanism for the formation of barium titanate fine particles on the basis of the above information.

\section{Discussion}

\subsection{Mechanism for the formation of barium} titanate fine particles

In this study, we found that the $\mathrm{Ba} / \mathrm{Ti}$ atomic ratio in the starting material significantly influenced the morphology and the fine structure of barium titanate fine particles, while temperature exerted only a slight influence because of the narrowness of the range from 70 to $150^{\circ} \mathrm{C}$. Therefore we propose that in the formation of barium titanate fine particles, the morphology is greatly influenced by the $\mathrm{Ba} / \mathrm{Ti}$ atomic ratio in the starting material.

The titanium tetrahydroxide used as a titanium source was a gel and thus a porous aggregate, as shown in Fig. 11. Barium hydroxide, however, dissolved completely in the solution under the conditions of this study because of its high solubility in warm water. We propose the following mechanism for the formation of barium titanate fine particles. The dissolved barium hydroxide is transported to the surface of the titanium tetrahydroxide gel in the solution, and is adsorbed on the surface. Then barium titanate forms via the reaction of titanium tetrahydroxide with barium hydroxide on the surface.

$$
\begin{gathered}
n \cdot \mathrm{Ti}(\mathrm{OH})_{4}+n \cdot \mathrm{Ba}(\mathrm{OH})_{2} \\
\rightarrow n \cdot \mathrm{BaTiO}_{3}+3 n \cdot \mathrm{H}_{2} \mathrm{O}
\end{gathered}
$$

This $n \cdot \mathrm{BaTiO}_{3}$ cluster is the supercritical embryo (hereafter, embryo) of barium titanate, and the number $n$ increases with a time. When the size of the cluster becomes larger than that of the critical nucleus, it becomes a stable supercritical nucleus (hereafter, nucleus) of barium titanate and begins to grow.

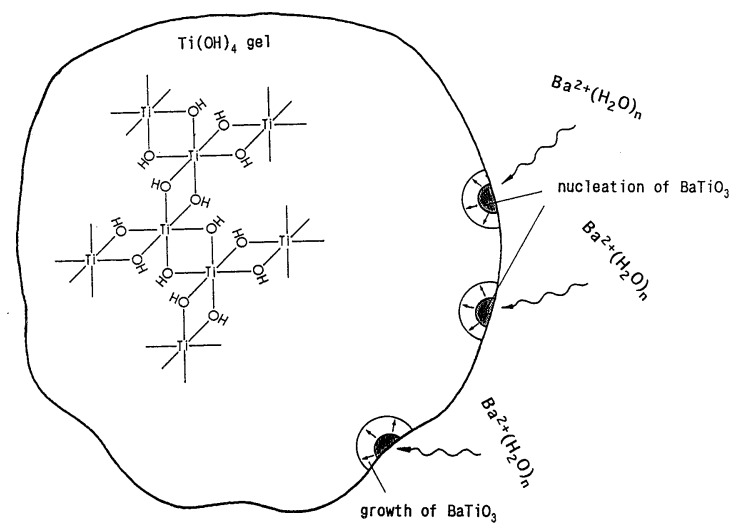

Fig. 11. The schematic diagram for the mechanism of the formation of barium titanate fine particles.
Under the formation conditions in this study (70$\left.150^{\circ} \mathrm{C}, 0.5 \mathrm{MPa}\right)$, the activation energy for the nucleation of barium titanate is higher than that for the growth of nuclei. Thus, as soon as barium titanate nuclei are formed, most of the barium ions in the solution are consumed in the growth of nuclei. On the assumption that the reaction constant for the nucleation is almost constant in the narrow temperature range from 70 to $150^{\circ} \mathrm{C}$, we can expect that the number of nuclei is also almost independent of temperature for a constant $\mathrm{Ba} / \mathrm{Ti}$ atomic ratio in the starting material. This was confirmed by the finding that the particle size is almost independent of temperature with a constant $\mathrm{Ba} / \mathrm{Ti}$ atomic ratio in the starting material.

The increase of the $\mathrm{Ba} / \mathrm{Ti}$ atomic ratio in the starting material corresponds to the increase of the concentration of barium hydroxide in the solution when the amount of titanium tetrahydroxide gel is constant. Under this condition, the number of barium titanate nuclei formed increases as the concentration of barium ions in the solution is increased, as shown in Fig. 12. Therefore the increase of the number of nuclei results in the decrease of the size of barium titanate fine particles, as shown in Fig. 12, because the amount of barium titanate formed is invariable.

In this study, when the concentration of barium ions in the solution became higher than a certain value $(=\mathrm{Ba} / \mathrm{Ti}$ atomic ratio of 20 , i.e., $1.3 \mathrm{~mol}$. $\mathrm{dm}^{-3}$ ), the particle size became almost constant at around $20 \mathrm{~nm}$. This means that the number of barium titanate nuclei formed became constant in the above-mentioned model. The solubility of barium hydroxide under a hydrothermal condition above $100^{\circ} \mathrm{C}$ becomes almost infinite, and thus barium ions were completely soluble under the formation conditions in this study. The number of barium ions which can adsorb on the surface of titanium tetrahydroxide gel increases with increasing $\mathrm{Ba} / \mathrm{Ti}$ atomic ratio. However, the number of sites for the adsorption of barium ions becomes constant at an equilibrium

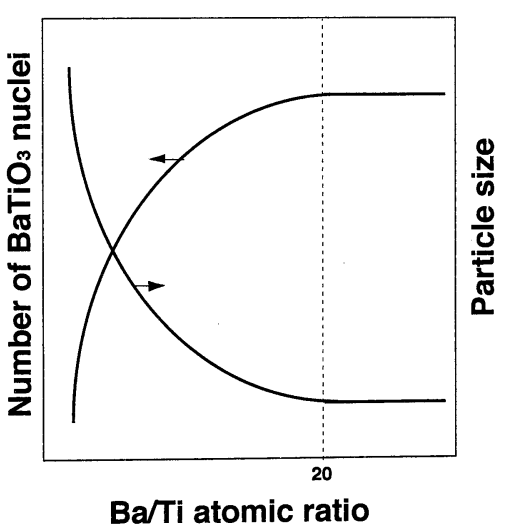

Fig. 12. The dependence of the number of barium titanate nuclei and the particle size on $\mathrm{Ba} / \mathrm{Ti}$ atomic ratio at a constant temperature. 
value. This is because the specific surface area of titanium tetrahydroxide gel is constant. Hence, the number of adsorbed barium ions also becomes constant at an equilibrium value. Under the formation conditions in this study, the equilibrium concentration of adsorbed barium ions is $1.3 \mathrm{~mol} \cdot \mathrm{dm}^{-3}(=\mathrm{Ba} /$ Ti atomic ratio of 20 ), and thus above this value the number of barium titanate nuclei can become constant. As a result, the particle size would also become constant.

In contrast, below the $\mathrm{Ba} / \mathrm{Ti}$ atomic ratio of 2 , the crystal structure of the particles becomes amorphous with decreasing $\mathrm{Ba} / \mathrm{Ti}$ atomic ratio in the starting material, and the amorphous phase changes to a titanium-rich barium titanate phase such as $\mathrm{Ba}_{2} \mathrm{Ti}_{9} \mathrm{O}_{20}$ upon thermal treatment at above $1000^{\circ} \mathrm{C}$. We believe that the following process causes the formation of the amorphous phase. The number of barium titanate nuclei decreases with decreasing concentration of barium hydroxide in the solution, and when the concentration of barium hydroxide in the solution becomes lower than a critical value, barium titanate embryos cannot grow into the critical nuclei because of the short-lived embryos and subsequently disappear.

In order to prepare barium titanate particles much smaller than $20 \mathrm{~nm}$, it is very important to use starting materials with much finer microstructure than that of titanium tetrahydroxide gel. The microstructure of titanium tetrahydroxide gel, however, is very porous in solution, and it is very difficult to prepare starting materials with much finer microstructure than that of titanium tetrahydroxide. It may be possible to prepare barium titanate particles with particle size of a few $\mathrm{nm}$ by the use of a completely dissolved titanium source.

\subsection{Lattice defects such as hydroxyl group and barium vacancies}

There were considerable amounts of $\mathrm{H}_{2} \mathrm{O}$ and $\mathrm{OH}^{-}$in barium titanate fine particles prepared in this study, and the amounts increased with decreasing particle size. These hydroxyl groups in the particles were comprised of the surface-adsorbed hydroxyl group and the lattice hydroxyl group. The amount of the surface-adsorbed hydroxyl group increased with decreasing particle size, as shown in Fig. 7 and by the results of TG measurements. ${ }^{22)}$ It is clear that the amount of the surface-adsorbed hydroxyl group per unit weight increases with decreasing particle size, assuming that the amount of the surface-adsorbed hydroxyl group per unit surface area is in-dependent of the particle size. This is because the surface area per unit weight increases with decreasing particle size. To compensate the charge of the surface-adsorbed hydroxyl group, there must be many barium vacancies in the region around the surface. We believe that these barium vacancies near the surface can be formed via the treatment with acetic acid $\left(6 \mathrm{~mol} \cdot \mathrm{dm}^{-3}\right)$.
In contrast, the concentration of the lattice hydroxyl group was independent of the particle size. This was supported by the results of TG measurements ${ }^{22)}$ as well as the results of the FT-IR measurements of the hydroxyl group (Fig. 7). The lattice hydroxyl group $\mathrm{OH}^{-}$in the barium titanate fine particles can exist as substitutional ions in the lattice position of oxygen $\mathrm{O}^{2-}$. Therefore the presence of cation vacancies is required in order to satisfy the electroneutrality condition in the particles. In fact, there are barium vacancies in these particles, and thus the electroneutrality condition using the lattice defects such as the lattice hydroxyl group and barium vacancies can be formulated as

$$
2\left[\mathrm{OH}_{0}{ }^{\circ}\right]=\left[\mathrm{V}_{\mathrm{Ba}}{ }^{\prime \prime}\right]
$$

Moreover when condition (3) is substituted into Eq. (2), the reaction for the formation of barium titanate can be indicated as

$$
\begin{aligned}
& \mathrm{Ti}(\mathrm{OH})_{4}+(1-x) \cdot \mathrm{Ba}(\mathrm{OH})_{2} \\
& \quad \rightarrow \mathrm{Ba}_{1-x} \mathrm{TiO}_{3-2 x} \mathrm{OH}_{2 x}+(3-2 x) \cdot \mathrm{H}_{2} \mathrm{O}
\end{aligned}
$$

These lattice hydroxyl groups may be distributed uniformly and randomly within a particle, because the particles show no XRD peak of an ordered lattice hydroxyl group (Fig. 2) and no lattice strain (Fig. 9).

On the basis of the above discussion, we can assume that a particle in this study is composed of the following two parts: (1) a defective surface layer including the surface-adsorbed hydroxyl group and (2) the bulk including the lattice hydroxyl group. Under this assumption, barium vacancies also must exist in two parts to satisfy each electroneutrality condition. In this study, the amounts of $\mathrm{OH}^{-}$and barium vacancies in the particles increased with decreasing particle size, as shown in Table 1 . We can explain this result using the above model that a particle can divide in two parts, because the volume fraction of the defective surface layer per unit weight increases with decreasing particle size. Quantitative discussion of this is given in another paper. ${ }^{22)}$

The lattice hydroxyl group is also thought to have originated from the hydroxyl group of the titanium tetrahydroxide used as the titanium source. Therefore it may be possible to prepare barium titanate without the lattice hydroxyl group by using starting materials without hydroxyl groups, e.g., $\mathrm{TiO}_{2}$.

\subsection{Crystal structure of the particles}

The crystal structure of the particles was assigned as cubic and their lattice constants $a$-axis were about $0.402 \mathrm{~nm}$ which is much greater than that $(0.4005$ $\mathrm{nm}$ ) obtained by the extrapolation of the $a$-axis of the cubic phase, which is stable above $T_{\mathrm{c}}$, to room temperature. ${ }^{26)}$ This indicated that the lattice can expand considerably. Barium titanate is an ionic crystal with ionic bonds such as $\mathrm{Ba}-\mathrm{O}$ and $\mathrm{Ti}-\mathrm{O}$ (although there are partly covalent bonds in $\mathrm{Ba}-\mathrm{O}$ and $\mathrm{Ti}-\mathrm{O}$ bonds). Hennings and Schreinemacher observed the enlargement of the barium titanate lattice with the presence of hydroxyl group in the lattice, and con- 
cluded that these lattice defects significantly influenced the Coulomb attractive forces which control ionic bonding strength. ${ }^{20)}$ These lattice defects can reduce the Coulomb attractive forces between ions. As a result, the bond length can increase, and thus the lattice can expand. The expansion of the barium titanate lattice in this study can also be explained using the model of Hennings and Schreinemacher.

In an ideal barium titanate single crystal, the crystal structure at around room temperature is tetragonal with tetragonality $(c / a)$ of 1.011 . However, the crystal structure of barium titanate fine particles prepared in this study was cubic with an expanded lattice, and the crystal structure and lattice constants were almost constant in spite of the considerable difference of the particle size (from 20 to $90 \mathrm{~nm}$ ). As one cause of these results, we consider the contribution of the lattice defects as well as of the particle size. In another paper, we discuss the formation of the cubic crystal structure of barium titanate due to the influence of the lattice defects such as the lattice hydroxyl group and the barium vacancy. ${ }^{27)}$

\section{Conclusion}

In this study, barium titanate fine particles were prepared hydrothermally using titanium tetrahydroxide gel and barium hydroxide as the starting materials. The morphology and the fine structure of the particles were found to depend greatly on the $\mathrm{Ba} / \mathrm{Ti}$ atomic ratio in the starting material while they were almost independent of temperature. Thus we proposed the mechanism for the formation of barium titanate fine particles, that nuclei of barium titanate were formed via the reaction of titanium tetrahydroxide gel with barium hydroxide dissolved in water, and then grew. Using this model we could explain the experimental results.

In these particles, there were many lattice defects in the form of hydroxyl groups and barium vacancies, and their concentration increased with decreasing particle size. We explained this result using the model that a particle can be divided into two parts: (1) a defective surface layer including the surface-adsorbed hydroxyl groups and (2) the bulk including the lattice hydroxyl group.

The crystal structure of barium titanate fine particles prepared in this study was cubic with an expanded lattice, and the crystal structure and lattice constants were almost constant regardless of the particle size. The expansion of the lattice could be explained by the weakening effect of the lattice defects on the ionic bonds in $\mathrm{Ba}-\mathrm{O}$ and $\mathrm{Ti}-\mathrm{O}$ bonds.

In this study, we prepared barium titanate fine particles with average particle size from 20 to $90 \mathrm{~nm}$. These particles included lattice defects such as hydroxyl groups and barium vacancies. Their crystal structure was cubic. The present preparation and characterization studies are the first step in the investigation of the size effect. We are now working to reduce the concentration of lattice defects by thermal treatment to remove the hydroxyl group, and to investigate the relationship between the crystal structure and the concentration of lattice defects.

Acknowledgements We thank Professor M. Yoshimura of Tokyo Institute of Technology for kindly allowing us the use of TG-DTA equipment.

\section{References}

1) K. Kinoshita and A. Yamaji, J. Appl. Phys., 47, 371-73 (1976).

2) G. Arlt, D. Hennings and G. de With, J. Appl. Phys., 58, 1619-25 (1985).

3) K. Ishikawa, K. Yoshikawa and N. Okada, Phys. Rev. B, 37, 5852-55 (1988).

4) K. Uchino, E. Sadanaga and T. Hirose, J. Am. Ceram. Soc., 72, 1555-58 (1989).

5) K. Oonishi, T. Morohashi and K. Uchino, Seramikkusu Ronbunshi, 97, 473-77 (1989).

6) K. Uchino, E. Sadanaga, K. Oonishi, T. Morohashi and H. Yamamura, "Ceramic Dielectrics-Ceramic Transactions, Vol. 8”, Am. Ceram. Soc. (1990) pp. 107-15.

7) J. Del Cerro, M. Mundi, C. Gallardo, J. M. Criado, F. J. Gotor and A. Bhalla, Ferroelectrics, 127, 1395-400 (1992).

8) T.-T. Fang, H.-L. Hsieh and F.-S. Shiau, J. Am. Ceram. Soc., 76, 1205-11 (1993).

9) T. Yamamoto, K. Urabe and H. Banno, Jpn. J. Appl. Phys., 32, 4272-76 (1993).

10) M. Kataoka, K. Suda, N. Ishizawa, F. Marumo, Y. Shimizugawa and K. Ohsumi, J. Ceram. Soc. Japan, 102, 213-16 (1994).

11) S. Wada, T. Suzuki and T. Noma, J. Mater. Res., 10, 306-11 (1995).

12) H. Ikawa, Seramikkusu, 27, 701-06 (1992).

13) H. Ikawa, "Dielectric Ceramics-Ceramic Transactions, Vol. 32", Am. Ceram. Soc. (1993) pp. 19-28.

14) S. Naka, F. Nakakita, Y. Suwa and M. Inagaki, Bull. Chem. Soc. Jpn., 47, 1168-71 (1974).

15） Y. Suyama and M. Nagasawa, J. Am. Ceram. Soc., 77, 60305 (1994).

16) R. Vivekanandan, S. Philip and T. R. N. Kutty, Mater. Res Bull., 22, 99-108 (1986).

17) K. Fukai, K. Hidaka, M. Aoki and K. Abe, Ceram. Int., 16, 285-90 (1990).

18) K. Kajiyoshi, N. Ishizawa and M. Yoshimura, J. Am. Ceram. Soc., 74, 369-74 (1991).

19) D. Hennings, G. Rosenstein and H. Schreinemacher, J. Eur. Ceram. Soc., 8, 107-15 (1991).

20) D. Hennings and S. Schreinemacher, J. Eur. Ceram. Soc., 9, 41-46 (1992).

21) P. K. Dutta and J. R. Gregg, Chem. Mater., 4, 843-46 (1992).

22) S. Wada, T. Suzuki and T. Noma, Jpn. J. Appl. Phys., in press.

23) K. Nakanishi, P. H. Solomon and N. Furutachi, "Infrared Absorption Spectroscopy”, Nankodo, Tokyo (1978) pp. 2934.

24) J. C. Slater, Phys. Rev., 78, 748-61 (1950).

25) W. G. Spitzer, R. C. Miller, D. A. Kleinman and L. E. Howarth, Phys. Rev., 126, 1710-21 (1962).

26) H. F. Kay and P. Vousden, Phil. Mag., 40, 1019-40 (1949).

27) S. Wada, T. Suzuki and T. Noma, submitted to J. Ceram. Soc. Japan. 